\title{
Craniofacial Polyostotic Fibrous Dysplasia Initially Diagnosed in a Primary Care Unit
}

\author{
Antonio Miguel Cruz-Ferreira, ${ }^{1,2 *}$ \\ ${ }^{1}$ Mealhada Primary Health Care Unit, Mealhada, Portugal \\ ${ }^{2}$ Faculty of Health Sciences, University of Beira Interior, Covilha, Portugal
}

Fibrous dysplasia (FD) is a non-malignant bone tumor that typically behaves as a slow and indolent growing mass lesion. We report the case of a female patient presenting with headache and facial deformity and later diagnosed with polyostotic fibrous dysplasia (PFD). A 29-year-old woman visited Mealhada Primary Health Care Unit complaining of headache, nasal congestion, and hyposmia for several weeks. She also presented with facial deformity and painful swelling of the upper left orbit. X-ray imaging revealed a suspicious opacity in the left frontal sinus and a right shift of the nasal septum. Computed tomography and bone scintigraphy later confirmed a tumor involving the ethmoid and frontal bone. The patient was referred to the neurosurgery and otorhinolaryngology departments of a central hospital and the suspected diagnosis of PFD was confirmed. A watchful waiting approach with regular imaging screenings was proposed and accepted by the patient, who is now free of symptoms and more acceptant of the benign condition of her tumor. With this case, we aim to make family physicians more aware of this rare but relevant condition that can be difficult to diagnose. FD is a rare but benign tumor that occurs mainly in adolescents and young adults. Symptoms depend on the location and type of the tumor and include facial deformity, vision changes, nasal congestion, and headache. No clear guidelines exist for its treatment, and options include monitoring the progression of the tumor, in addition to medical or surgical approaches.

Keywords: Fibrous Dysplasia of Bone; Bone Neoplasms; Primary Health Care

Received: November 13, 2017, Revised: December 6, 2017, Accepted: December 10, 2017

${ }^{*}$ Corresponding Author: Antonio Miguel Cruz-Ferreira https://orcid.org/0000-0002-9970-5259

Tel: +351-275329002, Fax: +351-275329099, E-mail: antonio.cruz.ferreira@ubi.pt 


\section{INTRODUCTION}

Fibrous dysplasia (FD) is a non-malignant bone tumor, accounting for $2.5 \%$ of all bone tumors, ${ }^{1)}$ caused by somatic activating mutations in the $\alpha$ subunit of the stimulatory $\mathrm{G}$ protein encoded by the gene GNAS. ${ }^{2)}$ In this condition, normal bone and marrow are replaced by fibrous tissue, and one bone (monostotic) or more (polyostotic fibrous dysplasia [PFD]) can be involved.

FD typically behaves as a slow and indolent growing mass lesion. Symptoms, which depend on the location and type of the tumor, are often unclear and the diagnosis can be delayed.

The PFD form is less prevalent (3:7 ratio) but affects the craniofacial region in $90 \%$ of cases, causing a multiplicity of symptoms, such as facial deformity, vision changes, hearing impairment, nasal congestion, local pain, and headache. ${ }^{2)}$ In many cases, patients are asymptomatic and the diagnosis is incidental. ${ }^{2)}$ Malignant change in sarcoma occurs in less than $1 \%$ of these tumors.

Because the natural course of the disease is variable, no categorical and uniformly accepted guidelines exist for the treatment of this condition. Monitoring the progression of the tumor, in addition to medical and/or surgical treatment, is accepted depending on the case. ${ }^{1)}$

We report a rare case of PFD involving the ethmoid and orbital surface of the frontal bone initially diagnosed in Mealhada Primary Health Care Unit.

\section{CASE REPORT}

A 29-year-old woman visited her new family physician complaining of headache, nasal congestion, and hyposmia for several weeks. She had no complaints of vision changes, skin lesions, paresthesia, numbness, or hearing impairment. The patient only had a history of rhinosinusitis with exacerbation in the spring and autumn, and was otherwise unremarkable. In the previous week, she had been using a nasal steroid spray and antihistamines without complete relief. Examination revealed asymmetry of the left eye and painful swelling of the upper left orbit was identified.

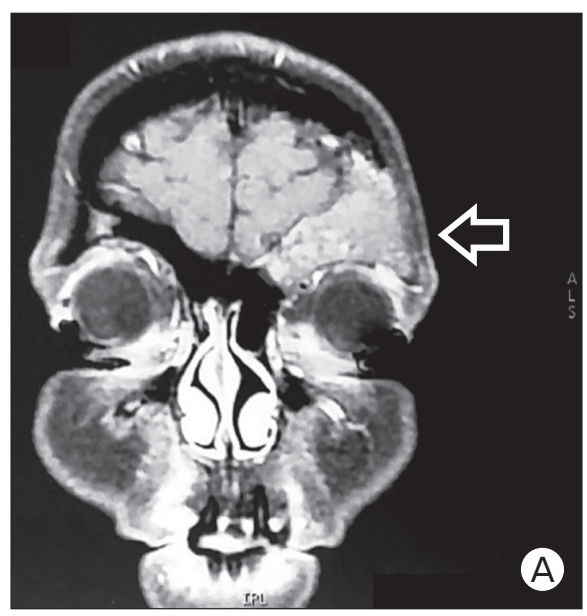

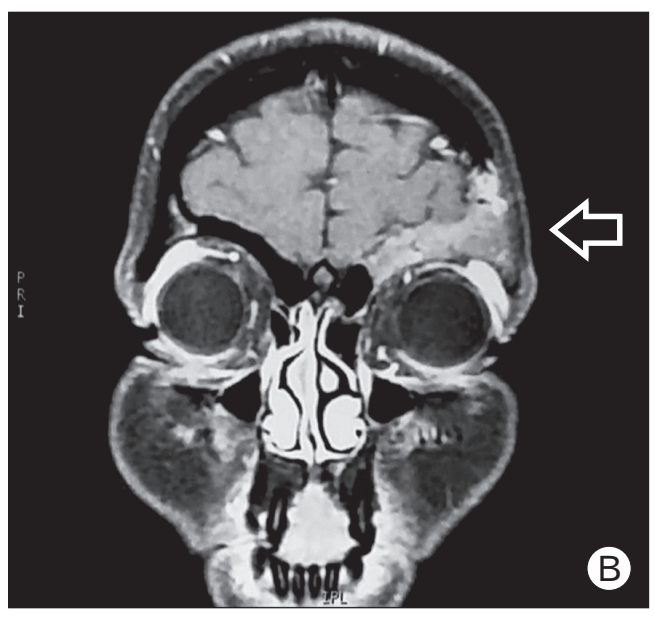

Figure 1. (A, B) Fibrous dysplasia. Magnetic resonance images of the patient, showing a lesion. Informed consent was obtained from the patient.
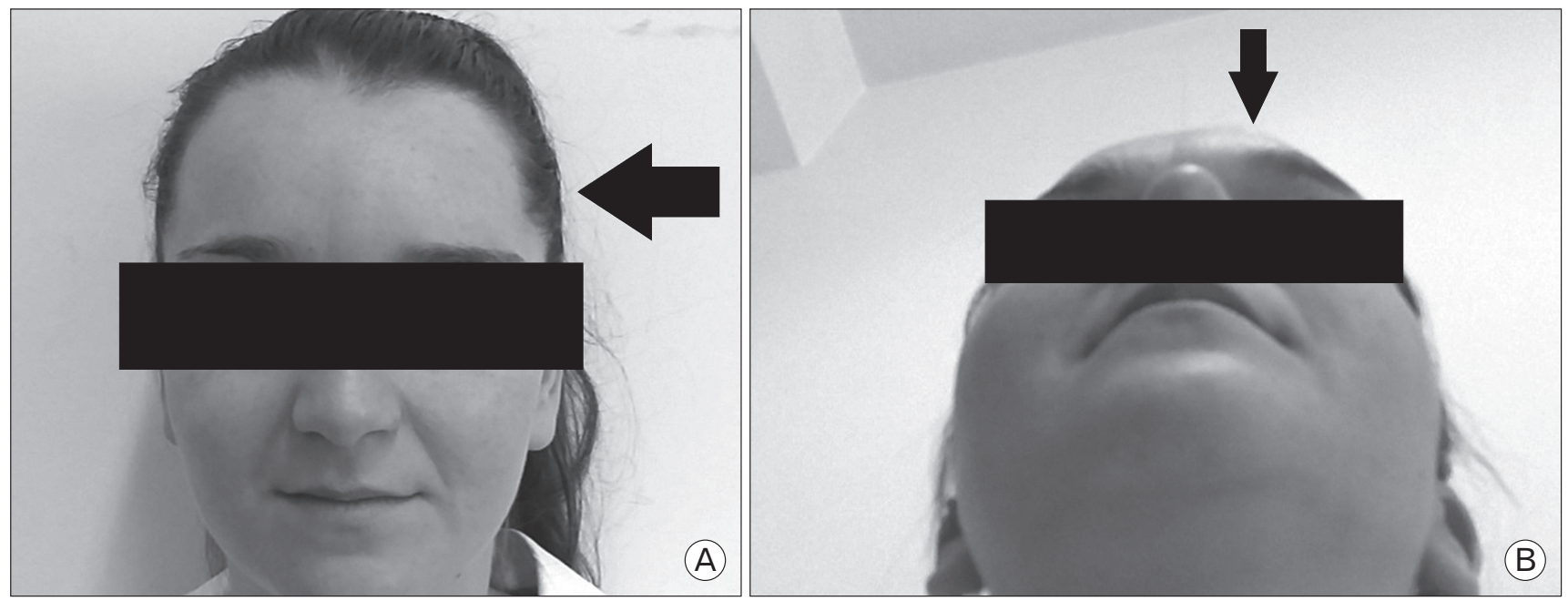

Figure 2. (A, B) Fibrous dysplasia. Photograph of the patient, showing slight facial deformity. Informed consent was obtained from the patient. 
These signs and symptoms were initially mistaken to be caused by sinusitis and an antibiotic was prescribed to treat that condition, as well as a non-steroid anti-inflammatory. However, because of the previously unidentified asymmetry of the left eye, an X-ray of the facial bones was requested. The X-ray revealed an opacity of the left frontal sinus and a right shift of the nasal septum, suggesting that more reliable assessment could be achieved with a computed tomography (CT) scan. The patient showed no signs of reduction in facial swelling despite improvement in other symptoms, and the CT scan revealed expansion of the anterior portion of the left frontal bone, with involvement of the orbital surface and the ethmoid bone, suggesting PFD.

With a probable diagnosis of PFD, the family physician referred the patient to both the neurosurgery and otorhinolaryngology departments of a central hospital.

After the initial assessment conducted by a neurosurgeon, magnetic resonance imaging and scintigraphy were performed. Both exams confirmed the presence of lesions in the orbital surface of both the frontal and ethmoid bones (Figure 1). Blood analysis excluded any endocrine abnormality. No biopsy was performed; however, the diagnosis of PFD was confirmed by a multidisciplinary team and a watchful waiting approach with periodical imaging assessments was recommended.

The patient currently presents no relevant symptoms, except for a slight deformity of the left eye (Figure 2) and the only medication taken is non-steroid anti-inflammatories in emergencies. She has accepted the benign condition of her tumor and, after an initial period of anxiety, has resumed her life without limitations.

Informed consent was obtained from the patient.

\section{DISCUSSION}

With this case we aim to make family physicians aware of this rare but relevant condition that can be difficult to diagnosis. FD is a rare benign tumor that occurs mainly in adolescents and young adults in the second and third decades of life. Males and females of all races are equally affected. ${ }^{4)}$ The thorax and extremities but also the face and neck bones can be affected, and two forms of presentation exist: monostotic and PFD. The latter is the rarest and involves craniofacial bones in $50 \%-$ $100 \%$ of cases. ${ }^{1)}$

Symptoms depend mainly on the site and type of the tumor, and its management is also variable, with no clearly accepted treatment guidelines. ${ }^{1)}$ Facial deformities, swelling, pain, vision changes, paresthesia, numbness, hearing impairment, headache, nasal congestion, and hyposmia are examples of frequent complaints these patients might report. Considering the symptoms, differential diagnosis typically includes rhinosinusitis, migraine, and brain injuries. ${ }^{2)}$ Imaging exams are crucial to help with the diagnosis.

Although rare and benign, in some severe cases the potential complications of untreated FD include bone deformity or fracture, vision or hearing loss, and malignant transformation, thus reinforcing the need for careful monitoring of its progression. ${ }^{1,4)}$

In 2012, Lee et al. ${ }^{2)}$ suggested a clinical guideline for the management of craniofacial FD. They agreed that the management of patients with FD should be based on the anatomic site and involvement of the tumor. For patients with PFD and craniofacial injuries and deformity, it is essential to monitor the growth of the tumor and to refer them to a maxillofacial and craniofacial surgeon if the patient is bothered by facial disfigurement.

When the sinuses are affected, symptoms such as nasal congestion, headache, facial pain, recurrent sinusitis, and hyposmia might be present. Nasal congestion and hyposmia seem to be correlated with the severity of the disease but not with pain or recurrent sinusitis. ${ }^{2)}$ As was attempted in our case, the management of these symptoms includes the use of nasal saline sprays, nasal steroid sprays, and antihistamines for patients with seasonal allergies, and antibiotics for patients with suspected bacterial sinus infections. ${ }^{2)}$ Furthermore, when a tumor grows in areas adjacent to the eyes, vision changes are a concern. As in our case, Lee et al. ${ }^{2)}$ also recommend referral to a multidisciplinary team in most cases more appropriate evaluation and treatment of these patients.

Regarding the non-surgical treatment of symptoms, most patients require pain treatment with non-steroid anti-inflammatories and/or bisphosphonates. Clinical trials have not yet demonstrated their efficacy in the treatment of pain in these patients. Although biphosphonates have also been suggested as therapeutic options for slowing the growth of craniofacial FD, additional studies are necessary to confirm their efficacy. ${ }^{2)}$

In conclusion, it is crucial to highlight that each PFD patient may present with distinct symptoms and a customized approach should be considered. It is of utmost importance that family physicians be made aware of this condition and they, in the unlikely event of a diagnosis (or suspected diagnosis), are able to conduct a comprehensive evaluation and request multidisciplinary involvement to achieve optimal care for patients.

\section{CONFLICT OF INTEREST}

No potential conflict of interest relevant to this article was reported.

\section{REFERENCES}

1. Numminen J, Rautiainen M. Polyostotic fibrous dysplasia of the mandible and bony nasal septum with intracranial expansion. Egypt J Ear Nose Throat Allied Sci 2016;17:49-51.

2. Lee JS, FitzGibbon EJ, Chen YR, Kim HJ, Lustig LR, Akintoye SO, et al. Clinical guidelines for the management of craniofacial fibrous dysplasia. Orphanet J Rare Dis 2012;7 Suppl 1:S2.

3. Sadeghi SM, Hosseini SN. Spontaneous conversion of fibrous dysplasia into osteosarcoma. J Craniofac Surg 2011;22:959-61.

4. Bhattacharya S, Mishra RK. Fibrous dysplasia and cherubism. Indian J Plast Surg 2015;48:236-48. 